PROCEEDINGS OF THE

AMERICAN MATHEMATICAL SOCIETY

Volume 140, Number 4, April 2012, Pages 1159-1171

S 0002-9939(2011)10999-7

Article electronically published on July 28,2011

\title{
QUASISYMMETRIC EXPANSIONS OF SCHUR-FUNCTION PLETHYSMS
}

\author{
NICHOLAS A. LOEHR AND GREGORY S. WARRINGTON
}

(Communicated by Jim Haglund)

\begin{abstract}
Let $s_{\mu}$ denote a Schur symmetric function and $Q_{\alpha}$ a fundamental quasisymmetric function. Explicit combinatorial formulas are developed for the fundamental quasisymmetric expansions of the plethysms $s_{\mu}\left[s_{\nu}\right]$ and $s_{\mu}\left[Q_{\alpha}\right]$, as well as for related plethysms defined by inequality conditions. The key tools for obtaining these expansions are new standardization and reading word constructions for matrices.
\end{abstract}

\section{INTRODUCTION}

For a partition $\lambda$, let $|\lambda|$ denote its size and $\chi^{\lambda}$ the corresponding irreducible character of the symmetric group $S_{|\lambda|}$. There are several natural ways to combine such characters to form new characters of a symmetric group. For example, the Kronecker or inner product of $\chi^{\lambda}$ and $\chi^{\mu}$ (defined only when $\left.|\lambda|=|\mu|\right)$ is a character of $S_{|\lambda|}$, the outer product of $\chi^{\lambda}$ and $\chi^{\mu}$ is a character of $S_{|\lambda|+|\mu|}$, while the inner plethysm of $\chi^{\lambda}$ and $\chi^{\mu}$ is a character of $S_{|\lambda||\mu|}[20, \S I .7,8]$. An important general problem in representation theory is to decompose these new characters into irreducible ones.

Using the characteristic map, which sends $\chi^{\lambda}$ to the Schur function $s_{\lambda}$, this problem can be approached via the theory of symmetric functions. Unfortunately, a fully explicit combinatorial solution is only known for the outer product, where the structure constants can be computed by the Littlewood-Richardson rule. In this paper we restrict our attention to the inner plethysm of $s_{\lambda}$ and $s_{\mu}$, denoted by $s_{\lambda}\left[s_{\mu}\right]$. The plethysm operation was introduced by Littlewood [17] in the context of compositions of representations of the general linear group. (The modern squarebracket notation reverses the order of his original notation $\{\mu\} \otimes\{\lambda\}$.) Plethysms have important connections to physics [24] and invariant theory [9]. Also, in the form of plethystic calculus, it has become a crucial computational tool for understanding diagonal harmonic modules and Macdonald's symmetric functions. One notable example can be found in [11]; see [18] for further references and details regarding plethysms.

Received by the editors May 25, 2010 and, in revised form, December 24, 2010

2010 Mathematics Subject Classification. Primary 05E05, 05E10.

The first author was supported in part by National Security Agency grant H98230-08-1-0045.

The second author was supported in part by National Security Agency grant H98230-09-1-0023.

(C)2011 American Mathematical Society Reverts to public domain 28 years from publication 
Numerous algorithms have been developed for computing the Schur expansion of a plethysm (most recently [5, 6, 25]), and several specific cases and extremal results have been obtained [2, 3, 4, 15, 22, 26]. But an explicit combinatorial formula for the full Schur expansion of an arbitrary plethysm $s_{\lambda}\left[s_{\mu}\right]$ has proved elusive. This is in sharp contrast to the expansion using monomial symmetric functions, which is easily described (see Section 3 ). In fact, there are several important symmetric functions for which we have combinatorial monomial expansions but lack combinatorially explicit Schur expansions. Examples include the modified Macdonald polynomials [13, 14, Lascoux-Leclerc-Thibon (LLT) polynomials [16] and (conjecturally) the image of a Schur function under the Bergeron-Garsia nabla operator [19.

All of the symmetric functions just mentioned, as well as the Schur functions themselves, have natural combinatorial expansions in terms of Gessel's fundamental quasisymmetric functions $Q_{\alpha}$ [12. These formulas arise because, in each case, there is a notion of standardization that converts "semistandard" objects appearing in the monomial expansion to "standard" objects. Part of the importance of these quasisymmetric expansions stems from two recently developed methods for converting quasisymmetric expansions of symmetric functions into Schur expansions. Assaf's method [1] uses a recursive algorithm to create dual equivalence graphs whose connected components correspond to terms in the Schur expansion. The second method, due to Egge and the authors [8, uses a modified version of the inverse Kostka matrix to produce explicit combinatorial formulas for the Schur coefficients that involve objects of mixed signs. Of course, for a Schur-positive symmetric function, all the negative objects must somehow cancel with some of the positive objects. The presence of these negative objects is undesirable, but there is significantly less cancellation encountered in converting from a fundamental quasisymmetric expansion to a Schur expansion than in converting from a monomial expansion to a Schur expansion 8 .

Our main result is the following explicit combinatorial formula for the expansion of Schur plethysms in terms of the fundamental quasisymmetric functions $Q_{\alpha}$.

Theorem 1.1. For all partitions $\mu, \nu$ with $|\mu|=a$ and $|\nu|=b$,

$$
s_{\mu}\left[s_{\nu}\right]=\sum_{A \in S_{a, b}(\mu, \nu)} Q_{\mathrm{Asc}(A)}
$$

Here, $S_{a, b}(\mu, \nu)$ is a certain set of "standard" $a \times b$ matrices depending on $\mu$ and $\nu$, and $\operatorname{Asc}(A)$ is a composition (or subset) determined by a new "reading word" construction for matrices (see Section 4 for precise definitions). Theorem 1.1 is proved by a standardization bijection on matrices, compatible with the new reading word, which is more subtle than the usual standardization operation on words or tableaux. The same technique furnishes combinatorial formulas for $s_{\mu}\left[Q_{\alpha}\right]$ and similar plethysms involving polynomials defined by inequality conditions. We note that Malvenuto and Reutenauer [21] have previously proved the $Q$-positivity of $s_{\mu}\left[Q_{\alpha}\right]$, a fact that implies, algebraically, the $Q$-positivity of $s_{\mu}\left[s_{\nu}\right]$ by plethystic addition rules. In contrast, we give direct bijective proofs of explicit combinatorial formulas for each of these plethysms. 
This paper is organized as follows. In Section 2, we review the necessary background on symmetric functions, quasisymmetric functions, and plethysms. Section 3 uses the definition of plethysm to obtain the (well-known) monomial expansion of $s_{\mu}\left[s_{\nu}\right]$, in which each monomial arises from a suitably weighted " $(\mu, \nu)$ matrix". Section 4 defines ascent sets and reading words for standard matrices. These lead to standardization and unstandardization bijections for matrices. These maps are used in Section 5 to establish Theorem 1.1 and related formulas. In Section 6, we give an example showing how the techniques in [8] can be used to calculate specific coefficients in the Schur expansions of Schur plethysms.

\section{Definitions And BACKGROUnd}

An integer partition is a weakly decreasing sequence $\mu=\left(\mu_{1} \geq \mu_{2} \geq \cdots \geq \mu_{k}\right)$, where each $\mu_{i}$ is an element of the positive integers $\mathbb{N}^{+}$. We say that $\mu$ is a partition of $n$, written $\mu \vdash n$, iff $\mu_{1}+\cdots+\mu_{k}=n$. The diagram of $\mu$ is the set

$$
\operatorname{dg}(\mu)=\left\{(i, j) \in \mathbb{N}^{+} \times \mathbb{N}^{+}: 1 \leq i \leq k, 1 \leq j \leq \mu_{i}\right\},
$$

which can be visualized as a set of unit squares in the first quadrant.

A semistandard tableau of shape $\mu$ with values in a totally ordered set $X$ is a function $T: \operatorname{dg}(\mu) \rightarrow X$ such that $T(i, j) \leq T(i, j+1)$ whenever $(i, j)$ and $(i, j+1)$ are in $\operatorname{dg}(\mu)$, and $T(i, j)<T(i+1, j)$ whenever $(i, j)$ and $(i+1, j)$ are in $\operatorname{dg}(\mu)$. The set of all semistandard tableaux of shape $\mu$ with values in $\{1,2, \ldots, N\}$ (resp. $\mathbb{N}^{+}$) is denoted $\operatorname{SSYT}_{N}(\mu)$ (resp. $\operatorname{SSYT}(\mu)$ ). The content monomial of a tableau $T \in \operatorname{SSYT}(\mu)$ is $x^{c(T)}=\prod_{(i, j) \in \operatorname{dg}(\mu)} x_{T(i, j)}$. The Schur symmetric polynomial in $N$ variables indexed by $\mu$ is

$$
s_{\mu}\left(x_{1}, \ldots, x_{N}\right)=\sum_{T \in \operatorname{SSYT}_{N}(\mu)} x^{c(T)} .
$$

The Schur symmetric function indexed by $\mu$ is the formal series

$$
s_{\mu}=\sum_{T \in \operatorname{SSYT}(\mu)} x^{c(T)}
$$

Taking $\mu=(m)$ gives the complete symmetric polynomial

$$
h_{m}\left(x_{1}, \ldots, x_{N}\right)=s_{(m)}\left(x_{1}, \ldots, x_{N}\right)=\sum_{1 \leq i_{1} \leq i_{2} \leq \cdots \leq i_{m} \leq N} x_{i_{1}} x_{i_{2}} \cdots x_{i_{m}}
$$

the complete symmetric function $h_{m}$ is defined analogously. It is known that the Schur polynomials $s_{\mu}\left(x_{1}, \ldots, x_{N}\right)$, as $\mu$ ranges over integer partitions with at most $N$ parts, form a basis for the $\mathbb{Q}$-vector space of symmetric polynomials in $x_{1}, \ldots, x_{N}$ (see, for example, [10]).

Given $k \in \mathbb{N}^{+}$, a composition of $k$ is a sequence $\alpha=\left(\alpha_{1}, \ldots, \alpha_{s}\right)$ of positive integers that sum to $k$. Compositions of $k$ correspond bijectively to subsets of $\{1,2, \ldots, k-1\}$ by mapping a composition $\alpha$ to the subset

$$
\operatorname{Gap}(\alpha)=\left\{\alpha_{1}, \alpha_{1}+\alpha_{2}, \alpha_{1}+\alpha_{2}+\alpha_{3}, \ldots, \alpha_{1}+\cdots+\alpha_{s-1}\right\} .
$$

The inverse bijection sends a subset $\left\{i_{1}<i_{2}<\cdots<i_{s}\right\}$ to the composition $\left(i_{1}, i_{2}-i_{1}, \ldots, i_{s}-i_{s-1}, k-i_{s}\right)$. In the following, we will sometimes identify the composition $\alpha$ with the subset $\operatorname{Gap}(\alpha)$. 
For a composition $\alpha$ of $k$, Gessel's fundamental quasisymmetric polynomial indexed by $\alpha$ is

$$
Q_{\alpha}\left(x_{1}, \ldots, x_{N}\right)=Q_{\operatorname{Gap}(\alpha)}\left(x_{1}, \ldots, x_{N}\right)=\sum_{\substack{1 \leq i_{1} \leq i_{2} \leq \ldots \leq i_{k} \leq N: \\ \text { strict ascents at } \\ \text { positions in Gap }(\alpha)}} x_{i_{1}} x_{i_{2}} \cdots x_{i_{k}} .
$$

More precisely, the condition on the subscript sequences in the sum is that they be weakly increasing and that $i_{j}<i_{j+1}$ for all $j \in \operatorname{Gap}(\alpha)$. Let $E(\alpha, N)=$ $E(\operatorname{Gap}(\alpha), N)$ be the set of sequences $\vec{\imath}=i_{1} i_{2} \cdots i_{k}$ satisfying these conditions and let the weight of such a sequence be $x_{i_{1}} \cdots x_{i_{k}}$. Then $Q_{\alpha}\left(x_{1}, \ldots, x_{N}\right)$ is the generating function for the weighted set $E(\alpha, N)$. We can define fundamental quasisymmetric functions similarly by dropping the requirement that $i_{k} \leq N$. We let $E(\alpha)=E(\operatorname{Gap}(\alpha))$ denote the associated set of subscript sequences.

Suppose $f\left(x_{1}, \ldots, x_{s}\right)$ and $g\left(x_{1}, \ldots, x_{t}\right)$ are polynomials such that $f$ is symmetric and $g=\sum_{i=1}^{s} m_{i}$, where each $m_{i}$ is a monic monomial involving the variables $x_{1}, \ldots, x_{t}$. (Note that the number $s$ of variables in $f$ is the same as the number of monomials appearing in the sum for $g$.) Then we can define the plethystic substitution of $g$ into $f$ by setting

$$
f[g]=f\left(m_{1}, \ldots, m_{s}\right) ;
$$

the ordering of the monomials $m_{i}$ is immaterial since $f$ is symmetric. If $g$ is symmetric in $x_{1}, \ldots, x_{t}$, so is $f[g]$. The same definition works if $f$ is a symmetric function and $g$ is a formal series that is a sum of countably many monic monomials. The definition of plethysm can be generalized to the case where $g$ is a polynomial or series that may involve negative terms, but we will only need the positive case just defined. See [18] for a more detailed discussion of plethysm.

Suppose $w=w_{1} w_{2} \cdots w_{n}$ and $y=y_{1} y_{2} \cdots y_{n}$ are two words of the same length using letters in a totally ordered alphabet $X$. We say that $w$ is lexicographically less than $y$, written $w<_{\operatorname{lex}} y$, iff there exists $k \leq n$ with $w_{i}=y_{i}$ for $1 \leq i<k$ and $w_{k}<y_{k}$. We write $w \leq_{\text {lex }} y$ iff $w=y$ or $w<_{\text {lex }} y$. One readily verifies that $X^{n}$, or any subset thereof, is totally ordered by $\leq_{\text {lex }}$.

\section{Monomial Expansion of $\boldsymbol{s}_{\boldsymbol{\mu}}\left[\boldsymbol{s}_{\boldsymbol{\nu}}\right]$}

Informally, $s_{\mu}\left[s_{\nu}\right]$ is the generating function for semistandard tableaux of shape $\mu$ using an alphabet consisting of all semistandard tableaux of shape $\nu$. To make this precise, we encode tableaux using reading words. Given any partition $\nu$, define the reading order of the cells of $\operatorname{dg}(\nu)$ by visiting the cells in the shortest row from left to right, then the cells in the next shortest row from left to right, and so on. The reading word of a tableau $T \in \operatorname{SSYT}(\nu)$, denoted $\operatorname{rw}(T)$, is obtained by listing the values $T(i, j)$ in reading order. For example, the tableau

$$
T=\begin{array}{|l|l|l|l|}
\hline 1 & 1 & 2 & 3 \\
\hline 2 & 2 & 3 & \\
\hline 3 & 3 & 4 \\
\hline
\end{array}
$$

of shape $\nu=(4,3,3)$ has reading word $\operatorname{rw}(T)=3342231123$.

If $\nu$ is fixed and known, each tableau $T \in \operatorname{SSYT}(\nu)$ is uniquely recoverable from its reading word. We can thereby identify $\operatorname{SSYT}(\nu)$ with the associated set of reading words, denoted $\mathcal{W}(\nu)$. More specifically, given $\nu=\left(\nu_{1}, \ldots, \nu_{s}\right)$, a word $a_{1} a_{2} \cdots a_{|\nu|}$ is in $\mathcal{W}(\nu)$ iff $a_{1} \leq a_{2} \leq \cdots \leq a_{\nu_{s}}, a_{\nu_{s}+1} \leq a_{\nu_{s}+2} \leq \cdots \leq a_{\nu_{s}+\nu_{s}-1}$, 
etc., and $a_{1}>a_{\nu_{s}+1}, a_{2}>a_{\nu_{s}+2}$, etc. The set of words $\mathcal{W}(\nu)$ is totally ordered by $\leq_{\text {lex }}$

Fix partitions $\mu$ and $\nu$ with $|\mu|=a$ and $|\nu|=b$. Let $\operatorname{SSYT}_{\mathcal{W}(\nu)}(\mu)$ be the set of semistandard tableaux of shape $\mu$ whose entries come from the totally ordered alphabet $\mathcal{W}(\nu)$. We can think of an object $T \in \operatorname{SSYT}_{\mathcal{W}(\nu)}(\mu)$ as a filling of $\operatorname{dg}(\mu)$ where each cell contains a tableau in $\operatorname{SSYT}(\nu)$ (see Figure 1 below). It will also be convenient to encode $T$ as an $a \times b$ matrix $A$, where row $i$ of $A$ is the reading word of the tableau in the $i$ th cell of $\operatorname{dg}(\mu)$ taken in reading order. Each row of $A$ must be a word satisfying the conditions indicated at the end of the last paragraph. In addition, the sequence of rows of $A$ must satisfy analogous conditions with $\nu$ replaced by $\mu$. For instance, if $\mu=\left(\mu_{1}, \ldots, \mu_{t}\right)$, the first $\mu_{t}$ rows of $A$ must form a lexicographically increasing sequence of words; row 1 of $A$ must be lex greater than row $\mu_{t}+1$ of $A$, etc. Matrices $A$ satisfying these conditions will be called $(\mu, \nu)$-matrices; let $M_{a, b}(\mu, \nu)$ be the set of all such matrices. The weight of $A \in M_{a, b}(\mu, \nu)$, denoted $\mathrm{wt}(A)$, is the product $\prod_{i, j} x_{A(i, j)}$. Combining (2.2) and (2.5), we deduce

$$
s_{\mu}\left[s_{\nu}\right]=\sum_{A \in M_{a, b}(\mu, \nu)} \operatorname{wt}(A) .
$$

Example 3.1. Let $\mu=(2,1)$ and $\nu=(2,2)$. The $(\mu, \nu)$-matrix

$$
C=\left[\begin{array}{llll}
4 & 5 & 3 & 4 \\
3 & 5 & 1 & 3 \\
4 & 6 & 2 & 2
\end{array}\right]
$$

contributes one term $x_{1} x_{2}^{2} x_{3}^{3} x_{4}^{3} x_{5}^{2} x_{6}$ to $s_{(2,1)}\left[s_{(2,2)}\right]$. $C$ encodes the "nested tableau" shown in Figure 1 .

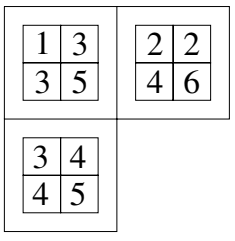

FiguRE 1. Nested tableau corresponding to one term of $s_{(2,1)}\left[s_{(2,2)}\right]$.

\section{Ascent SETS AND STANDARDiZATion For MATRICES}

To convert the monomial expansion in the last section to the quasisymmetric expansion of Theorem 1.1, we need to define notions of ascent sets and standardization for matrices. First, we review the more elementary versions of these concepts that apply to words and tableaux.

4.1. Standardization of Words. For $n \in \mathbb{N}^{+}$, let $S_{n}$ be the set of permutations of $\{1,2, \ldots, n\}$, i.e., words in which each symbol between 1 and $n$ occurs exactly once. Given any word $v=v_{1} v_{2} \cdots v_{n}$ with each $v_{i} \in \mathbb{N}^{+}$, we can produce its standardization $\operatorname{std}(v) \in S_{n}$ as follows. Suppose $v$ contains $k_{1}$ ones, $k_{2}$ twos, etc. Replace the ones in $v$ from left to right with $1,2, \ldots, k_{1}$; then replace the twos in $v$ from left to right with $k_{1}+1, k_{1}+2, \ldots, k_{1}+k_{2}$, and so on. Also, let $\operatorname{sort}(v)$ be 
the word obtained by sorting the letters of $v$ into weakly increasing order, and let $\operatorname{wt}(v)=\prod_{i=1}^{n} x_{v_{i}}=x_{1}^{k_{1}} x_{2}^{k_{2}} \cdots$. For example, given $v=311422413$, we have $\operatorname{std}(v)=612845937 \in S_{9} ; \quad \operatorname{sort}(v)=111223344 ; \quad \operatorname{wt}(v)=\operatorname{wt}(\operatorname{sort}(v))=x_{1}^{3} x_{2}^{2} x_{3}^{2} x_{4}^{2}$.

Given $w \in S_{n}$, the descent set of $w$ is $\operatorname{Des}(w)=\left\{i<n: w_{i}>w_{i+1}\right\}$. The inverse descent set of $w$ is $\operatorname{IDes}(w)=\operatorname{Des}\left(w^{-1}\right)$, which consists of all $j<n$ such that $j+1$ appears somewhere to the left of $j$ in $w$. We can identify these subsets of $\{1,2, \ldots, n-1\}$ with compositions of $n$ using the bijection in the introduction. For example, given $w=612845937$, we have $\operatorname{Des}(w)=\{1,4,7\}$ and $\operatorname{Des}\left(w^{-1}\right)=$ $\{3,5,7\}$. These subsets correspond to the compositions $(1,3,3,2)$ and $(3,2,2,2)$, respectively.

Let $X$ be the set of words of length $n$ in the alphabet $\mathbb{N}^{+}$. Let $Y$ be the set of pairs $(w, \vec{\imath})$, where $w \in S_{n}$ and $\vec{\imath}=i_{1} i_{2} \cdots i_{n}$ is a subscript sequence in $E(\operatorname{IDes}(w))$. One may check that the map $v \mapsto(\operatorname{std}(v), \operatorname{sort}(v))$ defines a bijection of $X$ onto $Y$. This bijection shows that

$$
\sum_{v \in X} \operatorname{wt}(v)=\sum_{w \in S_{n}} Q_{\operatorname{IDes}(w)}
$$

In turn, using well-known properties of the Robinson-Schensted-Knuth (RSK) algorithm and the $Q$-expansion of $s_{\lambda}$ (implicit in [12]), one can verify that

$$
\sum_{w \in S_{n}} Q_{\operatorname{IDes}(w)}=\sum_{\lambda \vdash n} f^{\lambda} s_{\lambda}
$$

where $f^{\lambda}$ is the number of standard tableaux of shape $\lambda$. This symmetric function is the Frobenius character of the left regular representation of the symmetric group $S_{n}$.

4.2. Standard Matrices and Ascent Sets. Let $M_{a, b}$ be the set of all $a \times b$ matrices with entries in $\mathbb{N}^{+}$. A matrix in $M_{a, b}$ is called standard iff its entries are $1,2, \ldots, a b$ in some order. Let $S_{a, b}$ be the set of standard $a \times b$ matrices. Given a standard matrix $A \in S_{a, b}$, we define its reading word $\mathrm{rw}(A)$ as follows. Scan the columns of $A$ from left to right. For each column index $k<b$, use the symbols in column $k+1$ of $A$ to determine a total ordering of the rows in which the row with the smallest symbol in column $k+1$ comes first, and the row with the largest symbol in column $k+1$ comes last. Write the symbols in column $k$ using the row ordering just determined. For the rightmost column, write the symbols in order from top to bottom. Given $a$ and $b$, we can recover a standard matrix $A$ from its reading word $\mathrm{rw}(A)$ by scanning the word backwards to recreate the columns of $A$ from right to left. Thus, the map rw $: S_{a, b} \rightarrow S_{a b}$ is a bijection.

The ascent set of the matrix $A$ is $\operatorname{Asc}(A)=\operatorname{IDes}(\operatorname{rw}(A))$. So $j \in \operatorname{Asc}(A)$ iff $j+1$ appears to the left of $j$ in $\operatorname{rw}(A)$. One may check that $j \in \operatorname{Asc}(A)$ iff either (a) $j+1$ appears in $A$ in a column to the left of the column containing $j$ or (b) $j$ and $j+1$ are both in the rightmost column of $A$, with $j+1$ in a higher row than $j$ or (c) $j$ and $j+1$ are in the same column (but not the rightmost column), and the number to the right of $j$ in its row exceeds the number to the right of $j+1$ in its row.

Example 4.1. The matrix

$$
A=\left[\begin{array}{cccc}
8 & 7 & 2 & 10 \\
6 & 1 & 9 & 4 \\
12 & 5 & 11 & 3
\end{array}\right] \in S_{3,4}
$$


has reading word

$$
\operatorname{rw}(A)=612871511921043
$$

and ascent set $\operatorname{Asc}(A)=\{3,4,5,7,10,11\}$. Given that $B \in S_{3,4}$ has reading word $\mathrm{rw}(B)=412869105273111$, we work backwards through the reading word to find that

$$
B=\left[\begin{array}{cccc}
4 & 6 & 2 & 3 \\
8 & 10 & 7 & 11 \\
12 & 9 & 5 & 1
\end{array}\right]
$$

4.3. Standardization of Matrices. Fix a matrix $C \in M_{a, b}$. Define the weight of $C$ to be $\operatorname{wt}(C)=\prod_{i=1}^{a} \prod_{j=1}^{b} x_{C(i, j)}$ and $\operatorname{sort}(C)$ to be the word $w_{1} w_{2} \cdots w_{a b}$ obtained by listing the entries of $C$ in weakly increasing order. Define $\operatorname{Comp}_{a, b}$ to be the set of "compatible" pairs $(A, \vec{\imath})$ for which $A \in S_{a, b}$ and $\vec{\imath}=i_{1} i_{2} \cdots i_{a b} \in$ $E(\operatorname{Asc}(A))$. Let the weight of such a pair be $\operatorname{wt}(A, \vec{\imath})=\operatorname{wt}(\vec{\imath})=\prod_{k=1}^{a b} x_{i_{k}}$.

Theorem 4.2. For all $a, b \in \mathbb{N}^{+}$, there are weight-preserving, mutually inverse bijections $S: M_{a, b} \rightarrow \operatorname{Comp}_{a, b}$ (standardization) and $U: \operatorname{Comp}_{a, b} \rightarrow M_{a, b}$ (unstandardization). Moreover,

$$
\sum_{C \in M_{a, b}} \operatorname{wt}(C)=\sum_{A \in S_{a, b}} Q_{\operatorname{Asc}(A)}=\sum_{w \in S_{a b}} Q_{\mathrm{IDes}(w)}=\sum_{\lambda \vdash a b} f^{\lambda} s_{\lambda} .
$$

Proof. Given a pair $(A, \vec{\imath}) \in \operatorname{Comp}_{a, b}$, we compute its unstandardization $U(A, \vec{\imath}) \in$ $M_{a, b}$ by replacing the unique copy of $j$ in $A$ by $i_{j}$, for $1 \leq j \leq a b$. For example,

$$
U\left(\left[\begin{array}{ccc}
1 & 4 & 6 \\
2 & 5 & 12 \\
3 & 9 & 11 \\
7 & 8 & 10
\end{array}\right], 111111222234\right)=\left[\begin{array}{lll}
1 & 1 & 1 \\
1 & 1 & 4 \\
1 & 2 & 3 \\
2 & 2 & 2
\end{array}\right]
$$

Given a matrix $C \in M_{a, b}$, its standardization is $S(C)=(\operatorname{std}(C)$, $\operatorname{sort}(C))$, where $\operatorname{std}(C)$ is the standard matrix obtained by the following algorithm. For each $k \geq 0$, let $N(k)$ be the number of symbols in $C$ that are less than or equal to $k$. Note that $N(0)=0$ and $N(k)=a b$ for large enough $k$. For any $i>0$, there are $N(i)-N(i-1)$ occurrences of $i$ in $C$. These $i$ 's will get renumbered by standard labels in the set $L_{i}=\{N(i-1)+1, N(i-1)+2, \ldots, N(i)\}$. The standardization algorithm operates as follows:

(1) Scan the rightmost column of $C$ from bottom to top, replacing each symbol $i$ as it is encountered by the largest unused label in $L_{i}$.

(2) For each $j$ from $b-1$ down to 1 , renumber the entries in column $j$ as follows. The previously renumbered column $j+1$ determines a total ordering of the rows, as in the definition of the ascent set. Scan the entries in column $j$ (according to the row ordering determined by column $j+1$ ) from the largest row to the smallest row. Replace each symbol $i$ as it is encountered by the largest unused label in $L_{i}$.

Example 4.3. An example of the standardization process is shown here:

$$
C=\left[\begin{array}{lllll}
1 & 1 & 3 & 3 & 5 \\
1 & 2 & 2 & 2 & 4 \\
2 & 2 & 3 & 3 & 3
\end{array}\right] \stackrel{S}{\mapsto}\left(A=\left[\begin{array}{ccccc}
1 & 3 & 10 & 12 & 15 \\
2 & 5 & 7 & 8 & 14 \\
4 & 6 & 9 & 11 & 13
\end{array}\right], 111222223333345\right) \text {. }
$$


Note that $N(0)=0, N(1)=3, N(2)=8, N(3)=13, N(4)=14$, and $N(5)=15$, so that $L_{1}=\{1,2,3\}, L_{2}=\{4,5,6,7,8\}, L_{3}=\{9,10,11,12,13\}, L_{4}=\{14\}$, and $L_{5}=\{15\}$. The columns of $A$ are filled in from right to left, with the scanning order for each column determined by the next column to the right. The last column is scanned from bottom to top; the fourth column from top to bottom; the third and second columns both in the order row 1 , row 3 , then row 2 ; and the first column from bottom to top. We have $\operatorname{rw}(A)=124563791011812151413$ and $\operatorname{Asc}(A)=\{3,8,13,14\}$. Observe that $S(C) \in \mathrm{Comp}_{3,5}$ and $U(S(C))=C$.

Continuing the proof, we must show that $S(C)$ is always in Comp $_{a, b}$. Since $A=$ $\operatorname{std}(C) \in S_{a, b}$, it is enough to verify that $\operatorname{sort}(C) \in E(\operatorname{Asc}(A))$. Write $\operatorname{sort}(C)=$ $i_{1} i_{2} \cdots i_{a b}$. This sequence is weakly increasing by definition. We see (by backwards induction on the column index) that the standardization algorithm creates the symbols in $A$ in the same order as they occur in a backwards scan of $\operatorname{rw}(A)$. Since the labels in each set $L_{i}$ are chosen from largest to smallest during this process, it follows that the subsequence of $\operatorname{rw}(A)$ consisting of the symbols in $L_{i}$ (read from left to right) is weakly increasing. Therefore, $\operatorname{Asc}(A) \subseteq\{N(1), N(2), \ldots\}$. Now, if $N(i-1)<k \leq N(i)$, then the symbol $k$ in $A$ was used to renumber one of the $i$ 's in $C$, and so $i_{k}=i$. Therefore, $k \in \operatorname{Asc}(A)$ implies $i_{k}<i_{k+1}$, proving that $\operatorname{sort}(C) \in E(\operatorname{Asc}(A))$.

One may routinely verify that $U \circ S=\mathrm{id}_{M_{a, b}}$. It is a little trickier to see that

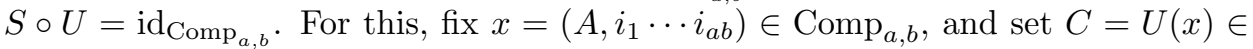
$M_{a, b}, x^{\prime}=S(C)=\left(A^{\prime}, i_{1}^{\prime} \cdots i_{a b}^{\prime}\right) \in \operatorname{Comp}_{a, b}$. Evidently, $i_{1} \cdots i_{a b}=\operatorname{sort}(C)=$ $i_{1}^{\prime} \cdots i_{a b}^{\prime}$. We must argue that $A^{\prime}=A$. Let $N(i)$ and $L_{i}$ be determined from $C$ as in the definition of $\operatorname{std}(C)$. When passing from $A$ to $C$, all labels in $L_{i}$ get replaced by occurrences of $i$. In turn, when passing from $C$ to $A^{\prime}$, these occurrences of $i$ get replaced by the standard labels in $L_{i}$. The key point to check is that this second replacement puts each $k \in L_{i}$ back in the same position in $A^{\prime}$ that $k$ occupied in $A$. The rules defining $S$ and $U$ are designed to force this property to hold. More precisely, in both of the reading words $\operatorname{rw}(A)$ and $\operatorname{rw}\left(A^{\prime}\right)$, the labels in each $L_{i}$ must occur as an increasing subsequence; this holds for $\operatorname{rw}(A)$ since $i_{1} \cdots i_{a b} \in E(\operatorname{Asc}(A))$, and it holds for $\operatorname{rw}\left(A^{\prime}\right)$ as shown in the previous paragraph. We can then prove that $A=A^{\prime}$ by backwards induction on the column index.

Thus $U$ and $S$ are inverses of each other, so both are weight-preserving bijections. Hence

$$
\sum_{C \in M_{a, b}} \operatorname{wt}(C)=\sum_{A \in S_{a, b}}\left(\sum_{\vec{\imath} \in E(\operatorname{Asc}(A))} \operatorname{wt}(\vec{\imath})\right) .
$$

The inner sum is precisely $Q_{\operatorname{Asc}(A)}=Q_{\operatorname{IDes}(\mathrm{rw}(A))}$. Since rw $: S_{a, b} \rightarrow S_{a b}$ is a bijection, (4.2) follows from (4.4) and (4.1).

\section{Proof of Theorem 1.1}

Given partitions $\mu \vdash a$ and $\nu \vdash b$, recall from Section 3 that $M_{a, b}(\mu, \nu)$ is the subset of $M_{a, b}$ consisting of $(\mu, \nu)$-matrices (which encode tableaux in $\operatorname{SSYT}_{\mathcal{W}(\nu)}(\mu)$ ). Let $S_{a, b}(\mu, \nu)=S_{a, b} \cap M_{a, b}(\mu, \nu)$ be the set of standard $(\mu, \nu)$-matrices. Let $\operatorname{Comp}_{a, b}(\mu, \nu)$ consist of all pairs $(A, \vec{\imath}) \in \operatorname{Comp}_{a, b}$ with $A \in S_{a, b}(\mu, \nu)$. Our goal is 
to prove Theorem 1.1, which states

$$
s_{\mu}\left[s_{\nu}\right]=\sum_{A \in S_{a, b}(\mu, \nu)} Q_{\operatorname{Asc}(A)}
$$

In light of Theorem 4.2, this equation will follow from (3.1) once we show that the maps $S$ and $U$ restrict to give bijections between $M_{a, b}(\mu, \nu)$ and $\operatorname{Comp}_{a, b}(\mu, \nu)$. This amounts to showing that $C \in M_{a, b}(\mu, \nu)$ iff $\operatorname{std}(C) \in S_{a, b}(\mu, \nu)$. Keeping in mind the definition of $(\mu, \nu)$-matrices, this last fact is an immediate consequence of the order-preservation properties appearing in the following lemma.

Lemma 5.1. Let $C \in M_{a, b}$ and $A=\operatorname{std}(C)$.

(a) For $1 \leq i \leq a$ and $1 \leq j<k \leq b, C(i, j) \leq C(i, k)$ iff $A(i, j)<A(i, k)$, and $C(i, j)>C(i, k)$ iff $A(i, j)>A(i, k)$.

(b) Let $C_{i}$ (resp. $A_{i}$ ) be row $i$ of $C$ (resp. A), viewed as a word of length $b$. For $1 \leq i<j \leq a, C_{i} \leq_{\operatorname{lex}} C_{j}$ iff $A_{i}<_{\text {lex }} A_{j}$, and $C_{j}<_{\text {lex }} C_{i}$ iff $A_{j}<_{\operatorname{lex}} A_{i}$.

Proof. It suffices to prove the forward directions of the four biconditional statements.

(a) Let $x=C(i, j)$ and $y=C(i, k)$. First, $x>y$ implies $A(i, j)>A(i, k)$ since every label in $L_{x}$ exceeds every label in $L_{y}$. Second, $x<y$ implies $A(i, j)<A(i, k)$ for the same reason. Third, $x=y$ implies $A(i, j)<A(i, k)$ since standardization creates the columns of $A$ from right to left, with occurrences of $x$ in $C$ replaced by elements of $L_{x}$ in decreasing order.

(b) Fix $i<j$ and assume $C_{i}<_{\operatorname{lex}} C_{j}$. This means that there exists $k \leq b$ with $C(i, s)=C(j, s)$ for $1 \leq s<k$, and $C(i, k)<C(j, k)$. The last condition implies that $A(i, k)<A(j, k)$. It then follows that $A(i, s)<A(j, s)$ for $1 \leq s \leq k$ by backwards induction on $s$, keeping in mind the standardization rules for two equal labels in the same column. In particular, $A(i, 1)<A(j, 1)$, so that $A_{i}<_{\text {lex }} A_{j}$. The same argument shows that $C_{j}<_{\operatorname{lex}} C_{i}$ implies $A_{j}<_{\operatorname{lex}} A_{i}$. Finally, consider the case $C_{i}=C_{j}$. Since $i<j, A(i, b)<A(j, b)$ follows by the standardization rule for the rightmost column. Then $A(i, s)<A(j, s)$ follows for $1 \leq s \leq b$ by backwards induction on $s$, as before. So $A(i, 1)<A(j, 1)$ and $A_{i}<_{\operatorname{lex}} A_{j}$.

Example 5.2. Take $\mu=(2,1)$ and $\nu=(2,2)$, and let

$$
C=\left[\begin{array}{llll}
4 & 5 & 3 & 4 \\
3 & 5 & 1 & 3 \\
4 & 6 & 2 & 2
\end{array}\right]
$$

as in Example 3.1. We compute

$$
A=\operatorname{std}(C)=\left[\begin{array}{llll}
7 & 11 & 5 & 9 \\
4 & 10 & 1 & 6 \\
8 & 12 & 2 & 3
\end{array}\right]
$$

and $S(C)=(A, 122333444556) \in \operatorname{Comp}_{3,4}(\mu, \nu)$. Furthermore,

$$
U(A, 122344556778)=\left[\begin{array}{llll}
5 & 7 & 4 & 6 \\
3 & 7 & 1 & 4 \\
5 & 8 & 2 & 2
\end{array}\right] \in M_{3,4}(\mu, \nu) .
$$


Example 5.3. Now take $\mu=(2,2)$ and $\nu=(2,1)$. We compute

$$
S\left(\left[\begin{array}{lll}
2 & 1 & 2 \\
3 & 1 & 2 \\
2 & 1 & 1 \\
2 & 1 & 1
\end{array}\right]\right)=\left(\left[\begin{array}{ccc}
9 & 3 & 10 \\
12 & 4 & 11 \\
7 & 1 & 5 \\
8 & 2 & 6
\end{array}\right], 111111222223\right),
$$

where both displayed matrices are $(\mu, \nu)$-matrices.

Example 5.4. Theorem 1.1 and the data in Table 1 lead to the formula

$h_{2}\left[h_{3}\right]=s_{(2)}\left[s_{(3)}\right]=Q_{33}+Q_{222}+Q_{24}+Q_{231}+Q_{42}+Q_{6}+Q_{321}+Q_{123}+Q_{141}+Q_{132}$.

TABLE 1. $Q$-expansion of $h_{2}\left[h_{3}\right]$.

\begin{tabular}{|c|c|c|c|c|c|}
\hline$A$ & {$\left[\begin{array}{lll}1 & 2 & 3 \\
4 & 5 & 6\end{array}\right]$} & {$\left[\begin{array}{lll}1 & 2 & 4 \\
3 & 5 & 6\end{array}\right]$} & {$\left[\begin{array}{lll}1 & 2 & 5 \\
3 & 4 & 6\end{array}\right]$} & {$\left[\begin{array}{lll}1 & 2 & 6 \\
3 & 4 & 5\end{array}\right]$} & {$\left[\begin{array}{lll}1 & 3 & 4 \\
2 & 5 & 6\end{array}\right]$} \\
\hline $\operatorname{rw}(A)$ & 142536 & 132546 & 132456 & 134265 & 123546 \\
\hline$\alpha$ & 33 & 222 & 24 & 231 & 42 \\
\hline \multirow{2}{*}{$A$} & {$\left[\begin{array}{lll}1 & 3 & 5\end{array}\right]$} & {$\left[\begin{array}{lll}1 & 3 & 6\end{array}\right]$} & {$[145]$} & {$\left[\begin{array}{lll}1 & 4 & 6\end{array}\right]$} & {$\left[\begin{array}{lll}1 & 5 & 6\end{array}\right]$} \\
\hline & {$\left[\begin{array}{lll}2 & 4 & 6\end{array}\right]$} & $245]$ & $\left.\begin{array}{lll}2 & 3 & 6\end{array}\right]$ & {$\left[\begin{array}{lll}2 & 3 & 5\end{array}\right]$} & {$\left[\begin{array}{lll}2 & 3 & 4\end{array}\right]$} \\
\hline $\operatorname{rw}(A)$ & 123456 & 124365 & 214356 & 213465 & 213564 \\
\hline$\alpha$ & 6 & 321 & 123 & 141 & 132 \\
\hline
\end{tabular}

Lemma 5.1 and matrix standardization provide the $Q$-expansions of many other plethysms, analogous to $s_{\mu}\left[s_{\nu}\right]$, whose monomial expansions are characterized by appropriately chosen inequalities. For instance, given $\mu \vdash a$ and $T \subseteq\{1,2, \ldots, b-1\}$ (corresponding to some composition $\alpha$ of $b$ ), we can obtain the quasisymmetric expansion of $s_{\mu}\left[Q_{\alpha}\right]$ as follows. Recall that $Q_{\alpha}$ is the generating function for weakly increasing sequences $\vec{\imath}=i_{1} i_{2} \cdots i_{b}$ with strict ascents at positions in $T$. Write $T=\left\{t_{1}<\cdots<t_{k}\right\}$ and define the reading word of $\vec{\imath}$ to be

$$
\operatorname{rw}(\vec{\imath})=i_{t_{k}+1} i_{t_{k}+2} \cdots i_{b} i_{t_{k-1}+1} i_{t_{k-1}+2} \cdots i_{t_{k}} \cdots i_{1} i_{2} \cdots i_{t_{1}} .
$$

Let $E^{\prime}(T)=E^{\prime}(\alpha)$ be the set of such reading words. One sees that membership in $E^{\prime}(T)$ is characterized by inequalities of the type appearing in Lemma 5.1(a). For example, $w=w_{1} \cdots w_{9} \in E^{\prime}(3213)$ iff $w_{1} \leq w_{2} \leq w_{3}$ and $w_{5} \leq w_{6}$ and $w_{7} \leq$ $w_{8} \leq w_{9}$ and $w_{1}>w_{4}>w_{6}$ and $w_{5}>w_{9}$. Now let $M_{a, b}(\mu, T)$ be the set of $a \times b$ matrices where each row is in $E^{\prime}(T)$, and the sequence of rows is the reading word of a tableau in $\operatorname{SSYT}_{E^{\prime}(T)}(\mu)$. Then (2.5) shows that $s_{\mu}\left[Q_{\alpha}\right]=\sum_{C \in M_{a, b}(\mu, T)} \mathrm{wt}(C)$. Define $S_{a, b}(\mu, T)=S_{a, b} \cap M_{a, b}(\mu, T)$ and $\operatorname{Comp}_{a, b}(\mu, T)=\left\{(A, \vec{\imath}): A \in S_{a, b}(\mu, T)\right\}$. Lemma 5.1 and matrix standardization show that

$$
s_{\mu}\left[Q_{\alpha}\right]=\sum_{A \in S_{a, b}(\mu, T)} Q_{\operatorname{Asc}(A)},
$$

as in the proof of Theorem 1.1. Other plethysms $f[g]$, where $f$ and $g$ are "defined by $\leq$ and $>$ conditions", can be handled in an entirely analogous way. 


\section{Schur Expansions From $Q$-EXPANSIONS}

There is a purely combinatorial way to convert the $Q$-expansion of any symmetric function to a Schur expansion [8]. We illustrate this technique here by determining the coefficient of $s_{(4,3,2)}$ in the plethysm $s_{(1,1,1)}\left[s_{(2,1)}\right]$. We begin by recalling the necessary details from [8].

A rim-hook is a contiguous border strip of a Ferrers diagram. A rim-hook tableau of shape $\lambda$ is a recursive tiling by rim-hooks of the Ferrers diagram of shape $\lambda$. A rim-hook tableau is special if every rim-hook begins in the first column, and it is flat if no rim-hook contains more than one box in the first column. The sign of a rim-hook spanning $r$ rows is $(-1)^{r-1}$, and the sign of a rim-hook tableau is the product of the signs of the rim-hooks in it. The content of a special rim-hook tableau is the composition determined by the lengths of its rim-hooks, read in order from the longest row of the diagram to the shortest row. There is at most one flat special rim-hook tableau of given shape $\lambda$ and content $\alpha$. Using notation stemming from the inverse Kostka matrix, we write $K_{n}^{*}(\alpha, \lambda)$ for the sign of such a flat special rim-hook tableau if one exists; we define $K_{n}^{*}(\alpha, \lambda)$ to be 0 if there is no such tableau.

Example 6.1. For $\lambda=(5,5,3,2)$ and $\alpha=(4,2,1,8)$, we have $K_{15}^{*}(\alpha, \lambda)=-1$. The relevant flat special rim-hook tableau is shown in Figure 2 .

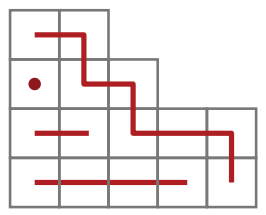

FiguRE 2. A flat special rim-hook tableau.

Let $f$ be a symmetric function with $Q$-expansion $f=\sum_{\alpha} y_{\alpha} Q_{\alpha}$ and Schur expansion $f=\sum_{\lambda} x_{\lambda} s_{\lambda}$. Then by Theorems 11 and 15 of [8], $x_{\lambda}=\sum_{\alpha} K_{n}^{*}(\alpha, \lambda) y_{\alpha}$.

As illustrated in Figure 3 , there are four flat special rim-hook tableaux of shape $(4,3,2)$. Taking into account their signs, we therefore have

$$
x_{432}=y_{432}-y_{414}-y_{252}+y_{216} \text {. }
$$
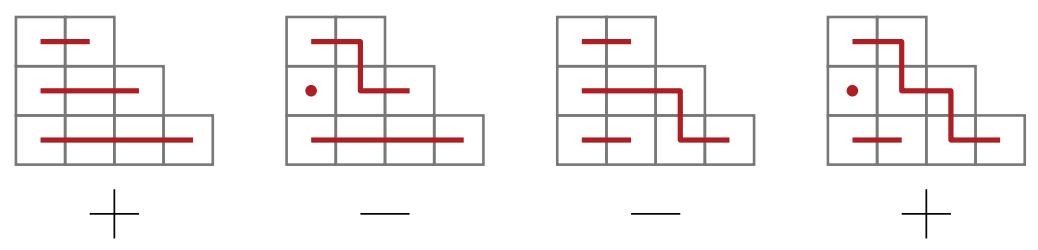

Figure 3. Flat special rim-hook tableaux of shape $(4,3,2)$.

So, to determine the coefficient of $s_{(4,3,2)}$ in the Schur expansion $s_{(1,1,1)}\left[s_{(2,1)}\right]$, we need only consider the standard fillings $A \in S_{3,3}((1,1,1),(2,1))$ whose ascent compositions $\alpha$ are in $\{432,414,252,216\}$. As listed in Table 2 , the numbers of such fillings are 5,2,2 and 0 , respectively. Incorporating this information into equation (6.1), we conclude that $y_{432}=5-2-2+0=1$. 
TABLE 2. Terms in the $Q$-expansion of $s_{(1,1,1)}\left[s_{(2,1)}\right]$ needed to compute the coefficient of $s_{(4,3,2)}$.

\begin{tabular}{|c|c|c|c|c|c|}
\hline$A$ & {$\left[\begin{array}{lll}8 & 1 & 4 \\
6 & 3 & 9 \\
5 & 2 & 7\end{array}\right]$} & {$\left[\begin{array}{lll}8 & 2 & 7 \\
6 & 3 & 9 \\
5 & 1 & 4\end{array}\right]$} & {$\left[\begin{array}{lll}8 & 3 & 9 \\
6 & 2 & 7 \\
5 & 1 & 4\end{array}\right]$} & {$\left[\begin{array}{lll}9 & 2 & 6 \\
8 & 1 & 4 \\
5 & 3 & 7\end{array}\right]$} & {$\left[\begin{array}{lll}9 & 6 & 7 \\
8 & 1 & 3 \\
5 & 2 & 4\end{array}\right]$} \\
\hline $\begin{array}{c}\operatorname{rw}(A) \\
\alpha\end{array}$ & $\begin{array}{c}856123497 \\
432\end{array}$ & $\begin{array}{c}586123794 \\
432\end{array}$ & $\begin{array}{c}568123974 \\
432\end{array}$ & $\begin{array}{c}895123647 \\
432\end{array}$ & $\begin{array}{c}859126734 \\
432\end{array}$ \\
\hline$A$ & {$\left[\begin{array}{lll}7 & 2 & 8 \\
6 & 1 & 4 \\
5 & 3 & 9\end{array}\right]$} & {$\left[\begin{array}{lll}8 & 3 & 9 \\
7 & 2 & 5 \\
6 & 1 & 4\end{array}\right]$} & {$\left[\begin{array}{lll}8 & 5 & 6 \\
4 & 2 & 9 \\
3 & 1 & 7\end{array}\right]$} & {$\left[\begin{array}{lll}9 & 4 & 5 \\
8 & 1 & 6 \\
3 & 2 & 7\end{array}\right]$} & \\
\hline $\begin{array}{c}\operatorname{rw}(A) \\
\alpha\end{array}$ & $\begin{array}{c}675123849 \\
414\end{array}$ & $\begin{array}{c}678123954 \\
414\end{array}$ & $\begin{array}{c}348512697 \\
252\end{array}$ & $\begin{array}{c}839412567 \\
252\end{array}$ & \\
\hline
\end{tabular}

Remark 6.2. The above technique can be used to give a combinatorial derivation of the well-known formula

$$
h_{2}\left[h_{n}\right]=\sum_{\substack{c=0 \\ c \text { even }}}^{n} s_{(2 n-c, c)} \quad(n \geq 1) .
$$

Many different proofs of this formula are known; the earliest proof appears to be that of Thrall [23]. See [20, I.8, Example 9] for instructions on how to derive (6.2) algebraically.

\section{REFERENCES}

1. S. Assaf. Dual equivalence graphs. I: A combinatorial proof of LLT and Macdonald positivity. arXiv:1005.3759

2. J. O. Carbonara, J. B. Remmel, and M. Yang. A combinatorial rule for the Schur function expansion of the plethysm $s_{\left(1^{a}, b\right)}\left[p_{k}\right]$. Linear and Multilinear Algebra, 39(4):341-373, 1995. MR:1365453 (97b:05164)

3. Luisa Carini and J. B. Remmel. Formulas for the expansion of the plethysms $s_{2}\left[s_{(a, b)}\right]$ and $s_{2}\left[s_{\left(n^{k}\right)}\right]$. Discrete Math., 193(1-3):147-177, 1998. Selected papers in honor of Adriano Garsia (Taormina, 1994). MR1661367(2000b:05129)

4. C. Carré. Plethysm of elementary functions. Bayreuth. Math. Schr., (31):1-18, 1990. MR:1056146 (91f:20013)

5. M. J. Carvalho and S. D'Agostino. Plethysms of Schur functions and the shell model. J. Phys. A, 34(7):1375-1392, 2001. MR1819938(2003a:05147)

6. Y. M. Chen, A. M. Garsia, and J. Remmel. Algorithms for plethysm. In Combinatorics and algebra (Boulder, Colo., 1983), volume 34 of Contemp. Math., pages 109-153. Amer. Math. Soc., Providence, RI, 1984. MR777698(86f:05010)

7. Ö. Egecioglu and J. B. Remmel. A combinatorial interpretation of the inverse Kostka matrix, Linear and Multilinear Algebra, 26(1-2):59-84, 1990. MR.1034417(90m:05011)

8. E. Egge, N. Loehr, and G. Warrington. From quasisymmetric expansions to Schur expansions via a modified inverse Kostka matrix. European J. Combin. 31(8):2014-2027, 2010. MR 2718279

9. H. O. Foulkes. Concomitants of the quintic and sextic up to degree four in the coefficients of the ground form. J. London Math. Soc., 25:205-209, 1950. MR0037276 (12:236e)

10. W. Fulton. Young Tableaux; with applications to representation theory and geometry, volume 35 of London Mathematical Society Student Texts. Cambridge University Press, Cambridge, 1997. MR1464693 (99f:05119) 
11. A. M. Garsia and J. Haglund. A proof of the $q, t$-Catalan positivity conjecture. Discrete Math., 256(3):677-717, 2002. LaCIM 2000 Conference on Combinatorics, Computer Science and Applications (Montreal, QC). MR1935784(2004c:05207)

12. Ira M. Gessel. Multipartite $P$-partitions and inner products of skew Schur functions. In Combinatorics and algebra (Boulder, Colo., 1983), volume 34 of Contemp. Math., pages 289-317. Amer. Math. Soc., Providence, RI, 1984. MR777705 (86k:05007)

13. J. Haglund. A combinatorial model for the Macdonald polynomials. Proc. Natl. Acad. Sci. USA, 101(46):16127-16131 (electronic), 2004. MR2114585 (2006e:05178)

14. J. Haglund, M. Haiman, and N. Loehr. A combinatorial formula for Macdonald polynomials. J. Amer. Math. Soc., 102:2690-2696, 2005. MR2141666 (2006g:05223b)

15. T. M. Langley and J. B. Remmel. The plethysm $s_{\lambda}\left[s_{\mu}\right]$ at hook and near-hook shapes. Electron. J. Combin., 11(1):Research Paper 11, 26 pp. (electronic), 2004. MR2035305 (2004j:05128)

16. Alain Lascoux, Bernard Leclerc, and Jean-Yves Thibon. Ribbon tableaux, Hall-Littlewood functions and unipotent varieties. Sém. Lothar. Combin., 34:Art. B34g, approx. 23 pp. (electronic), 1995. MR1399754 (98m:05195)

17. D. E. Littlewood. Invariant theory, tensors and group characters. Philos. Trans. Roy. Soc. London. Ser. A., 239:305-365, 1944. MR0010594(6:41c)

18. N. Loehr and J. Remmel. A computational and combinatorial exposé of plethystic calculus. J. Algebraic Combin., 33(2):163-198, 2011. MR.2765321

19. Nicholas A. Loehr and Gregory S. Warrington. Nested quantum Dyck paths and $\nabla\left(s_{\lambda}\right)$. Int. Math. Res. Not. IMRN, (5):Art. ID rnm 157, 29, 2008. MR2418288 (2009d:05257)

20. I. G. Macdonald. Symmetric functions and Hall polynomials. Oxford Mathematical Monographs. Oxford University Press, New York, second edition, 1995. With contributions by A. Zelevinsky, Oxford Science Publications. MR.1354144 (96h:05207)

21. Claudia Malvenuto and Christophe Reutenauer. Plethysm and conjugation of quasi-symmetric functions. Discrete Math., 193(1-3):225-233, 1998. Selected papers in honor of Adriano Garsia (Taormina, 1994). MR1661370(2000a:05211)

22. S. P. O. Plunkett. On the plethysm of $S$-functions. Canad. J. Math., 24:541-552, 1972. MR.0294526 (45:3596)

23. R. M. Thrall. On symmetrized Kronecker powers and the structure of the free Lie ring. Amer. J. Math., 64:371-388, 1942. MR0006149(3:262d)

24. Brian G. Wybourne. Symmetry principles and atomic spectroscopy. Wiley-Interscience [A division of John Wiley \& Sons], New York-London-Sydney, 1970. Including an appendix of tables by P. H. Butler. MR 0421392 (54:9396)

25. Mei Yang. An algorithm for computing plethysm coefficients. In Proceedings of the 7th Conference on Formal Power Series and Algebraic Combinatorics (Noisy-le-Grand, 1995), Discrete Math. 180:391-402, 1998. MR1603696 (99d:05088)

26. Mei Yang. The first term in the expansion of plethysm of Schur functions. Discrete Math., 246(1-3):331-341, 2002. Formal power series and algebraic combinatorics (Barcelona, 1999). MR $1887494(2003 \mathrm{e}: 05143)$

Department of Mathematics, Virginia Tech, Blacksburg, Virginia 24061

E-mail address: nloehr@vt.edu

Department of Mathematics and Statistics, University of Vermont, Burlington, VerMONT 05401

E-mail address: gwarring@cems.uvm.edu 